\title{
A New Acylated Stilbene Glycoside from Acanthopanax brachypus
}

\author{
Hao-bin $\mathrm{Hu}^{\dagger+*}$ and Jun Fan ${ }^{\div-}$

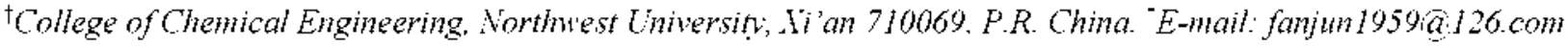 \\ -College of Chemistry and Chemical Engineering, Longdong Lniversity, Oingung 745000, P.R. China \\ Received August 3. 2008, Accepted December 11. 2008
}

Key Wonds: Acanthopanax brachypus, Araliaceae. Acylated stilbene glycoside, Phenolic glycoside

The Acanthopanax genus belonging to the Araliaceae family includes 37 species around the world. are widely distributed in Korea. Japan. China and the far-eastern region of Russia. 26 species and 18 varieties grow in mainland China. The root and stem bark of these plants have been clinically used for a long time as a tonic and sedative. as well as for the treatment of rheumatism. diabetes chronic bronchitis. hy'pertension, anti-stress and ischemic heart disease. and gastric ulcer. ${ }^{3-6}$ As a endangered slrub in the wild due to overharvesting and loss of habitat through deforestation. Acanthopanar brachypus Harms is distributed in a narrow geographical area. most in the loess plateau of the northwest of China. ${ }^{-8}$ Research indicates that the seeds of $A$. brachwpus contains many kinds of microelements indispensable to human body. can relax women's menopause sýndrome and exhibit immunostimulatory and anticancer activities, and its rhizomatic extracts has also been successfully used for inhibition of the various allergic responses in China and Korea. ${ }^{9-11}$ Nowadays, the other parts of this plant such as the roots. leaves and flowers are employed for various therapeutic purposes. ${ }^{12-14}$ To date. however, the research mainly concentrated on the reproductive biology and ecology, there has been few studies on the chemical composition and biological activity. Only six compounds, ${ }^{15.16}$ syringaresinol diglucoside syringin sucrose $\beta$-sitosterol. fatty acid and olefine acid have been previously isolated from this plant. With the aim to find active principle and provide the reference for quality control and effective utilization of Acanthopanax species, ${ }^{1,-z]}$ further phytochemical investigation led to the isolation of one new acylated stilbene glycoside. namely

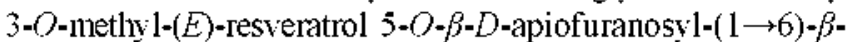
$D-\left[2^{t \prime}-(E)\right.$-cinnamoyl]-glucopy ranoside 1 , together with three known phenolic glycosides. namely $(E)$-resveratrol 3-O-a$D$-glucopyranoside 2. (E)-3-hydroxyanethole 3-O- $\beta$-D-glucopyranoside 3 and orcinol $O-\beta$ - $D$-glucopyranoside 4 . were isolated for the first time from the stem bark of $A$. brachipus. In the present communication we describe the isolation and structural elucidation of these compounds.

Compound 1 as a pale yellowish powder from $\mathrm{MeOH}$. its positive-ion HR-FAB-MS exhibited the molecular ion at $m: z$ $667.2381[\mathrm{M}+\mathrm{H}]^{+}$. corresponding to the molecular formula $\mathrm{C}_{35} \mathrm{H}_{38} \mathrm{O}_{13}$. which indicated 17 degrees of unsaturation. The fragmention ions at $m: z 37,535,405.243$ and 131 indicated that 1 contains a hexose-pentose and a cinnamoyl moiety. The UV spectrum exhibited absorptions at 220,308 and 324 rum. suggesting the presence of a conjugated aromatic system. The IR spectrum apart from hỵdrosyl $\left(3+29 \mathrm{~cm}^{-1}\right)$, aromatic rings (1600. 1512. $\left.1456 \mathrm{~cm}^{-1}\right)$ and glycosidic bond (1058 $\mathrm{cm}^{-1}$ ) absorption bands. showed an additional carbonyl absorption bands at 1698,1632 and $970 \mathrm{~cm}^{-1}$. indicating the presence of an ester group conjugated with a rans-double bond. ${ }^{23.24}$ The ${ }^{1} \mathrm{H}$ NMR spectnum of 1 showed the presence of two anomeric protons. resonating at $\delta_{\mathrm{H}}+.91$ (1H. d. $J=7.5$ $\mathrm{Hz}$ ) and $5.10(\mathrm{lH}, \mathrm{d}, J=2.4 \mathrm{~Hz})$. in addition to the signals for a 1,3.5-trisubstituted aromatic ring at $\delta_{\mathrm{H}} 6.70(1 \mathrm{H}$, br s). 6.40 $\left(\mathrm{IH}\right.$. br s) and $6.63\left(\mathrm{lH}\right.$. br s), one methoxy group at $\delta_{\mathrm{H}} 3.8 \mathrm{I}$ $(3 \mathrm{H} . \mathrm{s})$. a parc-disubstituted aromatic ring at $\delta_{\mathrm{H}} 7.43(2 \mathrm{H}, \mathrm{d}, J$ $\left.=8.5 \mathrm{~Hz}, \mathrm{H}-2^{\prime}, 6^{\prime}\right)$ and $6.88\left(2 \mathrm{H}, \mathrm{d}, J=8.5 \mathrm{~Hz} . \mathrm{H}-3^{\prime} .5^{\prime}\right)$, and two trans-olefinic protons at $\partial_{\mathrm{H}} 6.85 / 7.0 \mathrm{I}$ (each $\mathrm{H}$, d. $J=16.0$ $\mathrm{Hz}) .^{25}$ These spectra were consistent with those published for $(E)$-resveratrol ${ }^{2-t^{2}}$ and $(E)$-resveratrol 3-O- $\beta$ - $D$-glucopyranoside. $^{28,29}$ indicating that 1 had an $(E)$-resveratrol aglycon moiety. In the NMR and DEPT spectrum of 1. the signals of one anomeric carbon $\left(\delta_{C} 101.3\right)$. four methines $\left(\delta_{C} 76.8 .74 .5\right.$. 71.3 and 76.9$)$ and one methylene $\left(\delta_{0}, 68.2\right)$ indicated that the
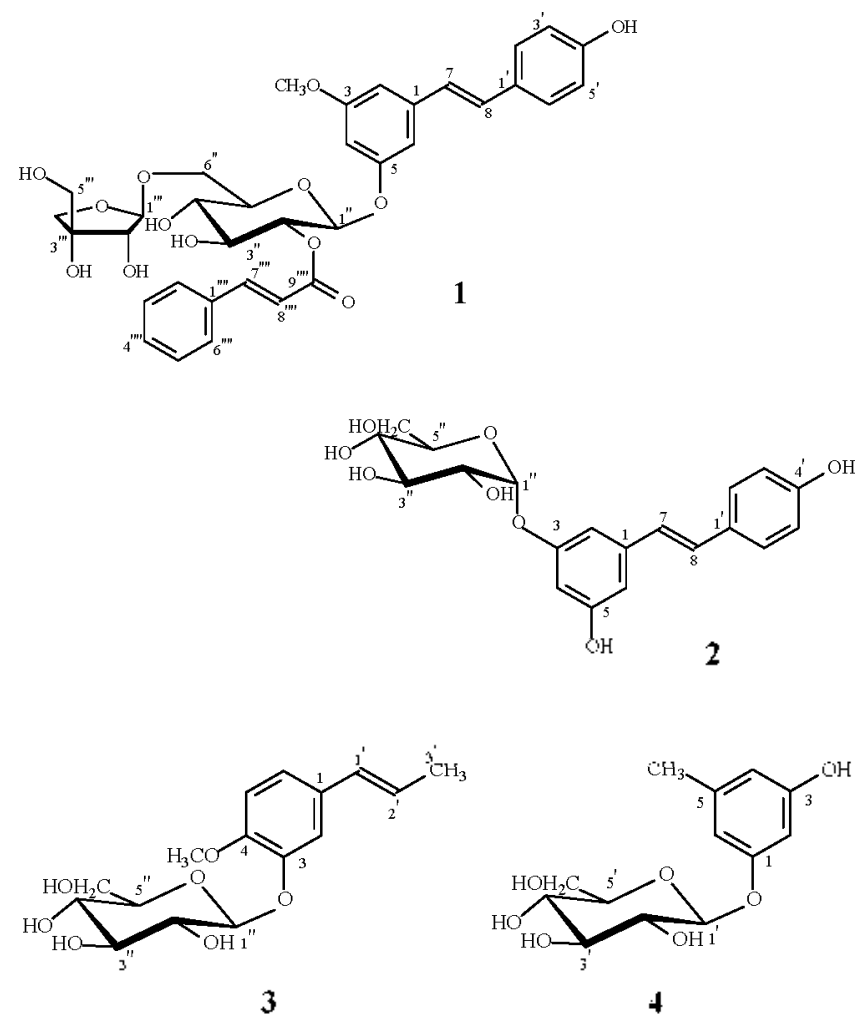

Figure 1. The structure of compounds 1-4 isolated from Acanthopanax brachypus. 
hexose of the disaccharide moiety was $D$-glucopyranose with $\beta$-anomeric configuration on the basis of the coupling constant $(J=7.5 \mathrm{~Hz})$ of the ${ }^{1} \mathrm{H}$ NMR signal at $\delta_{\mathrm{H}} 4.91$, and the pentose was $D$-apiofiranose with $\beta$-configuration based on the anomeric proton $\left[\partial_{\mathrm{H}} 5.10(\mathrm{lH} . \mathrm{d} . J=2 .+\mathrm{Hz})\right]$ and anomeric carbon $\left(\partial_{C}\right.$ $109.5)$, one methine $\left(\delta_{0}, 78.2\right)$, two methylenes $\left(\delta_{C} 74.8\right.$ and $65.1)$ and one quaternary carbon $(\delta, 80.7)$ signals. ${ }^{3.9}$ The remaining signals at $\dot{y}_{\mathrm{H}} 6.30 / 7.23(2 \mathrm{H}, \mathrm{d}, J=16.0 \mathrm{~Hz}) .7 .48(2 \mathrm{H}, \mathrm{d}, J$ $=7.6 \mathrm{~Hz}) .7 .3 \mathrm{l}(2 \mathrm{H}$. dd. $J=7.0$ and $7.6 \mathrm{~Hz}) .7 .35(1 \mathrm{H} . \mathrm{t} . J=7.0$ $\mathrm{Hz}$ ). and $\delta_{c} 16+8$ indicated a trans-cinnamoyl moiety in 1 . In the FAB-MS spectrum of 1 . the significant ion peaks at $m: z$ 537 [M+H-cinnamoyl] $]^{-} .535[\mathrm{M}+\mathrm{H} \text {-apiosyl] }]^{+}, 405[\mathrm{M}+\mathrm{H}$-cinnamoyl-apiosyl] ${ }^{+} .243$ [M+H-cinnimoyl-apiosyl-glucosyl] $]^{-} 131$ [cinnamoyl] $]^{-}$also indicated the presence of cinnamoyl. glucosyl and apiosyl groups in 1 . This conclusion was further evidenced by the detection of trans-cinnamic acid, $D$-glucose and $D$-apiose (in the ratio of $1: 1$, determined by $c o$-TLC with authentic sample and GC-MS analy sis ${ }^{31}$ ) after acid hydrolysis.

By comparing ${ }^{13} \mathrm{C}$ NMR spectral data of 1 with 5-O-methyl(E)-resveratrol 3-O- $\beta$ - $D$-apiofuranosyl- $(1 \rightarrow 6)-\beta-D$-glucopy ranoside. ${ }^{3 ?}$ indicated that the cimnamoyl moiety was attached to the $\mathrm{C}-2^{\prime \prime}$ of glucose as this carbon signal was shifted downfield ${ }^{33-36}$ by 1.90 ppm. while the $\mathrm{C}-1$ " and $\mathrm{C}-3^{\prime \prime}$ signals shifted upfield by 1.20 and $3.40 \mathrm{ppm}$, respectively. The terminal $D$-apiosyl group connected $\mathrm{C}-6$ " of the glucosyl group by a ( 1 $\rightarrow 6$ )-O-gly'cosidic linkage. because $C-6^{\prime \prime}$ was shifted downfield about 5 ppm. The glucosyl group connected C-5 of the aglycon through an $O$-glycosidic bond because of the downfield chemical shift of $\mathrm{C}-5$. The methoxyl group was linked to $\mathrm{C}-3$ as its shifted downfield. These aforementioned conclusions were also supported by the correlatons of $\mathrm{H}-2^{\prime \prime} / \mathrm{C}-9^{\prime \prime \prime}$. $\mathrm{H}-\mathrm{I}^{\prime \prime \prime} /$ $\mathrm{C}-6 ", \mathrm{H}-6 " / \mathrm{C}-1^{\prime \prime} . \mathrm{H}-1^{\prime \prime} / \mathrm{C}-5 . \mathrm{H}-\mathrm{OCH} / \mathrm{C}-3, \mathrm{H}-\mathrm{I}^{\prime \prime} / \mathrm{H}-6, \mathrm{H}-2 " /$ $\mathrm{H}-4^{\prime \prime} . \mathrm{H}-3^{\prime \prime} / \mathrm{H}-5^{\prime \prime}$ and $\mathrm{H}-\mathrm{OCH} / \mathrm{H}-2$ in the HMBC and ROESY spectra (Fig. 2). On the basis of the foregoing studies. the structure of 1 was established as 3-O-methyl- $(E)$-resveratrol $5-O-\beta$ - $D$-apiofuranosyl- $(1 \rightarrow 6)-\beta-D-\left[2^{\prime \prime}-(E)\right.$-cimnamoyl $]$-glucopyranoside.

Compound 2 was elucidated as (E)-resveratrol 3-O- $\alpha-D$ glucopyranoside by comparing its NMR data with those reported in the literature. ${ }^{3 ? 38}$

Compound 3 was hydrolyzed in acid solution to give $D$-glucose detected by TLC. further characterized as $(E)$ 3-hydroxyanethole 3-O-B-D-glucopyranoside by detailed spectroscopic analysis and comparisons of its physical and spec-

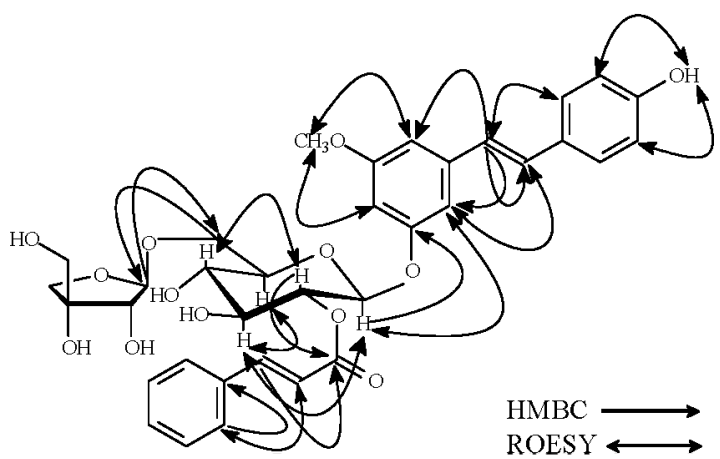

Figure 2. Significant HMBC $\left(\hat{0}^{-\hat{3}} J_{I-H}\right)$ and ROESY correlations for compound 1 . tral data with reported values. ${ }^{39}$

Compound 4 exhibited NMR and melting point data identical to values in the literature, ${ }^{+(i .+1]}$ thus it was established as orcinol $O-\beta-D$-glucopy ranoside.

To our knowledge. compound 2, 3 and 4 were also isolated for the first time from the stem bark of . $A$. brachypus.

\section{Experimental Section}

General experimental procedures. Melting points were obtained on an X-4 digital nucro-melting point apparatus and

Table 1. ${ }^{1} \mathrm{H}$ and ${ }^{13} \mathrm{C}$ NMR assignments of compounds 1 in (400 and $100 \mathrm{MHz}, J_{\mathrm{Hz}}$, in CD, OD, TMS)

\begin{tabular}{|c|c|c|c|}
\hline No. & $\delta_{c}(\mathrm{DEPT})$ & $\delta_{\mathrm{H}}\left(J_{\mathrm{Hz}}\right)$ & $\mathrm{HMBC}(\mathrm{H} \rightarrow \mathrm{C})$ \\
\hline \multicolumn{4}{|c|}{ Aglycon moiety } \\
\hline 1 & $141.5(\mathrm{C})$ & & \\
\hline 2 & $107.3(\mathrm{CH})$ & $6.70(1 \mathrm{H}$, br s $)$ & $1,3,4,6, \alpha$ \\
\hline 3 & $160.1(C)$ & & \\
\hline 4 & $104.0(\mathrm{CH})$ & $6.40(1 \mathrm{H}$, br s $)$ & $2,3,5,6$ \\
\hline 5 & $159.3(C)$ & & \\
\hline 6 & $107.8(\mathrm{CH})$ & $6.63(1 \mathrm{H}$, br s $)$ & $1,2,4,5, \alpha$ \\
\hline 7 & $126.8(\mathrm{CH})$ & $6.85(1 \mathrm{H}, \mathrm{d}, 16.0)$ & $2,6,1^{\prime}, \beta$ \\
\hline 8 & $129.8(\mathrm{CH})$ & $7.01(1 \mathrm{H}, \mathrm{d}, 160)$ & $1,2^{\prime}, 6^{\prime}, \alpha$ \\
\hline 9 & $55.9\left(\mathrm{CH}_{3}\right)$ & $3.81(3 \mathrm{H}, \mathrm{s})$ & 3 \\
\hline $1^{\prime}$ & $131.6(\mathrm{C})$ & & \\
\hline $2^{\prime}$ & $128.7(\mathrm{CH})$ & $7.43(1 \mathrm{H}, \mathrm{d}, 8.5)$ & $3,4, \beta$ \\
\hline $3^{\prime}$ & $115.4(\mathrm{CH})$ & $6.88(1 \mathrm{H}, \mathrm{d}, 8.5)$ & $14^{\prime}$ \\
\hline $4^{\prime}$ & $158.7(\mathrm{C})$ & & \\
\hline 5 & $115.4(\mathrm{CH})$ & $6.88(1 \mathrm{H}, \mathrm{d}, 8.5)$ & $l^{\prime} \cdot 4$ \\
\hline $6^{\prime}$ & $128.7(\mathrm{CH})$ & $7.43(1 \mathrm{H}, \mathrm{d}, 8.5)$ & $4^{\prime}, 5^{\prime}, \beta$ \\
\hline \multicolumn{4}{|c|}{ Sugar moiety } \\
\hline $1^{\prime \prime}$ & $101.3(\mathrm{CH})$ & $4.91(1 \mathrm{H}, \mathrm{d}, 7.5)$ & $5,2^{\prime \prime}$ \\
\hline $2^{\prime \prime}$ & $76.8(\mathrm{CH})$ & $4.53(1 \mathrm{H}, \mathrm{dd}, 9.5,7.5)$ & $1^{\prime \prime}, 3^{\prime \prime}, 9^{\prime \prime \prime}$ \\
\hline $3^{\prime \prime}$ & $74.5(\mathrm{CH})$ & $3.41(1 \mathrm{H}, \mathrm{t}, 9.5)$ & $2^{\prime \prime}, 4^{\prime \prime}$ \\
\hline $4^{\prime \prime}$ & $71.3(\mathrm{CH})$ & $3.45(1 \mathrm{H}, \mathrm{m})$ & $3^{\prime \prime}, 5^{\prime \prime}$ \\
\hline $5^{\prime \prime}$ & $76.9(\mathrm{CH})$ & $3.58(1 \mathrm{H}, \mathrm{m})$ & $4^{\prime \prime}, 6^{\prime \prime}$ \\
\hline $6^{t+}$ & $68.2\left(\mathrm{CH}_{2}\right)$ & $\begin{array}{l}3.98(1 \mathrm{H}, \mathrm{dd}, 11.5,1.8) \\
3.71(1 \mathrm{H}, \mathrm{dd}, 11.5,5.0)\end{array}$ & $5^{\prime \prime}, 1^{m+1}$ \\
\hline $1^{m+n}$ & $109.5(\mathrm{CH})$ & $5.10(1 \mathrm{H}, \mathrm{d}, 2.4)$ & $6^{n}, 3^{n+1}, 4^{n+1}$ \\
\hline $2^{\prime \prime \prime}$ & $78.2(\mathrm{CH})$ & $3.74(1 \mathrm{H}, \mathrm{d}, 2.4)$ & $5^{\prime \prime \prime}$ \\
\hline $3^{\prime \prime \prime}$ & $80.7(C)$ & & \\
\hline $4^{m+1}$ & $74.8\left(\mathrm{CH}_{2}\right)$ & $3.87 / 3.61(2 \mathrm{H}, \mathrm{d}, 9.1)$ & $3^{t+}, 5^{m}$ \\
\hline $5^{\prime \prime \prime}$ & $65.1\left(\mathrm{CH}_{2}\right)$ & $3.39 / 3.36(2 \mathrm{H}, \mathrm{d}, 10.0)$ & $4^{\prime \prime \prime}$ \\
\hline \multicolumn{4}{|c|}{ Ester moiety } \\
\hline $1^{m+t}$ & $133.8(\mathrm{C})$ & & \\
\hline $2^{\prime \prime \prime}$ & $130.2(\mathrm{CH})$ & $7.48(1 \mathrm{H}, \mathrm{d}, 7.6)$ & $1^{m \prime \prime}, 4^{m \prime \prime}, 6^{m \prime \prime}, 7^{m \prime \prime}$ \\
\hline $3^{n+t}$ & $128.1(\mathrm{CH})$ & $7.31(1 \mathrm{H}, \mathrm{dd}, 7.6,7.0)$ & $1^{n t+}=2^{n+t)}, 5^{m+t}$ \\
\hline $4^{m+t}$ & $129.2(\mathrm{CH})$ & $7.35(1 \mathrm{H}, \mathrm{t}, 7.0)$ & $2^{n+1}, 6^{n+1+1}$ \\
\hline $5^{\prime \prime \prime}$ & $128.1(\mathrm{CH})$ & $7.31(1 \mathrm{H}, \mathrm{dd}, 7.6,7.0)$ & $1^{m \prime \prime}, 3^{\prime \prime \prime}$ \\
\hline $6^{\prime \prime \prime}$ & $130.2(\mathrm{CH})$ & $7.48(1 \mathrm{H}, \mathrm{d}, 7.6)$ & $1^{m \prime \prime}, 4^{\prime \prime \prime}, 7^{m \prime \prime}$ \\
\hline $7^{\prime t+t}$ & $144.5(\mathrm{CH})$ & $7.23(1 \mathrm{H}, \mathrm{d}, 16.0)$ & $2^{n t+}=8^{m+t}=9^{m+t}$ \\
\hline $8^{\prime \prime \prime}$ & $117.4(\mathrm{CH})$ & $6.30(1 \mathrm{H}, \mathrm{d}, 16.0)$ & $1^{m \prime \prime}, 7^{m m}, 9^{m \prime \prime}$ \\
\hline $9^{\prime \prime \prime}$ & $164.8(C)$ & & \\
\hline
\end{tabular}

${ }^{a}$ Assigniments were confirmed by ID and 2D NMR methods. 
uncorrected. Optical rotations were measured on a PerkinElmer 341 digital polarimeter. UV spectra were recorded on a Shimadzu UV-300 spectrophotometer. IR spectra were recorded on IMPACT-400 FTIR spectrometer with $\mathrm{KBr}$ pellets. ${ }^{\mathrm{l}} \mathrm{H}$ $(400 \mathrm{MHz}) .{ }^{13} \mathrm{C}(100 \mathrm{MHz})$ and $2 \mathrm{D} \mathrm{NMR}$ spectra were determined on a Bruker DRX-400 spectrometer using tetramethylsilane as the internal standard. Chemical shifts $(\delta)$ are given in ppm and coupling constants $(J)$ in $\mathrm{Hz}$. Mass spectra were obtained on VG Alto Spec-3000 mass spectrometer. GC-MS: GC6890NMSD5973N: HP-5 MS fused silica capillary column (30 m $\times$ $0.25 \mathrm{~mm}$. film thinkness $0.25 \mu \mathrm{m}$ ). Column chromatography was carried out on silica gel (100-200, 200-300 mesh) and Sephadex LH-20 (25-100 $\mu \mathrm{m})$. Thin-layer chromatography (TLC) was performed on precoated sillica gel plates $\left(\mathrm{GF}_{354}\right)$. and spots were visualized under UV light ( 254 and $365 \mathrm{~nm}$ ) by spraying proper colored reagent.

Plant materials. The stem bark of A. brachypts were collected in August of 2007. from Qingyang of Gansu Province. and were identified by Prof. Xiao-qiang Guo. Department of LifeSciences. Longdong University. A voucher specimen (10732) was deposited in the Herbarium of the Department of LifeSciences. Longdong University . People's Republic of China.

Extraction and isolation. The air-dried and pulverized stem barks of $A$. brachypus $(5.0 \mathrm{~kg})$ were extracted three times with $95 \% \mathrm{EtOH}(15 \mathrm{~L} \times 7 \mathrm{~d}$. each time) at room temperature, and then the extracts were combined and concentrated under reduced pressure at $60^{\circ} \mathrm{C}$ to yield $242 \mathrm{~g}$ of a brown viscous residue. The EtOH extract was suspended in water and partitioned successively with $n$-hexane. EtOAc and $n-\mathrm{BuOH}$. The EtOAcsoluble extract ( $188 \mathrm{~g}$ ) was subjected to silica-gel column and eluated with $n$-hexane. $\mathrm{CHCl}_{3} . \mathrm{CHCl}_{3}-\mathrm{Me} \mathrm{CHO}_{2} \mathrm{C}$ (from $5: 1$ to 1:5. $\mathrm{v} / \mathrm{v}$ ). $\mathrm{Me} \mathrm{e}_{-} \mathrm{CO}$ and $\mathrm{Me} \mathrm{e}_{-} \mathrm{CO}-\mathrm{MeOH}$ (from $10: 1$ to $1: 10 . \mathrm{v} / \mathrm{v}$ ) linear gradient. the eluted fractions were examined by analytical TLC and similar fractions were pooled together to give 18 main fractions $\left(A_{1}-A_{18}\right)$. Fraction $A_{5}$ was rechromatographed over silica-gel column eluting with $\mathrm{CHCl}_{3}-\mathrm{MeOH}$ $(4: 1 . \mathrm{v} / \mathrm{v})$ and further purified by preparative TLC $(\mathrm{MeOH} /$ $\mathrm{CHCl}_{3} / n$-hexane. 1:5:2. v/v) to afford compound $+(24 \mathrm{mg})$. Fraction $\mathrm{A}_{8}$ was chromatographed over silica-gel column with a gradient mixture of $\mathrm{CHCl}_{3}-\mathrm{MeOH}$ (from $8: 1$ to $1: 8 . \%$ ) to give two subtraction $\left(A_{81}\right.$ and $A_{82}$ ). Subfraction $A_{81}$ was chromatographed on Sephade. LH-20 with MeOH- $\mathrm{H}_{2} \mathrm{O}$ (1:1. $\mathrm{v} / \mathrm{v})$ to afford compound $3(40 \mathrm{mg})$. Subfraction $\mathrm{A}_{82}$ was rechromatographed on silica-gel column with EtOAc-MeOH $(5: 2 . v / v)$ to afford compound $2(16 \mathrm{mg})$. Fraction $A_{13}$ was further purified on silica-gel column eluting stepwise with $\mathrm{CHCl}_{3}-\mathrm{MeOH}$ (from $5: 1$ to $1: 8, \mathrm{v} / \mathrm{v}$ ), and then on Sephadex $\mathrm{LH}-20$ eluting with $\mathrm{MeOH}$ to obtain compound 1 (3I mg).

3-O-Methyl-(E)-resveratrol 5-O- $\beta-D$-apiofuranosyl- $(1 \rightarrow$ 6)- $\beta-D-\left[2^{\prime \prime}-(E)\right.$-cinnamoyl]-glucopyranoside (1): Pale yellowish pouder $(\mathrm{MeOH})$, mp. $232-233^{\circ} \mathrm{C},[\alpha]_{\bar{c}}^{\circ}-32.9^{\circ}$ (c 0.14 . $\mathrm{MeOH})$ : UV $\lambda_{\text {max }}^{\mathrm{MeOH}}(\log \varepsilon): 220(4.24), 308(4.46)$ and 324 (4.56) nm: $\mathbb{R}{ }^{\prime}{ }_{\max }^{\mathrm{kBr}}: 3429,1698,1632,1600,1512,1456,1278$. 1140. 1058. $970 \mathrm{~cm}^{-1}$ : HR-FAB-MS (positive mode) $m z$ : $667.2381[\mathrm{M}+\mathrm{H}]^{-}$(calculated for $\mathrm{C}_{35} \mathrm{H}_{3} \mathrm{O}_{13}, 667.2391$ ): FAB-MS (positive mode) $m: z: 667[\mathrm{M}+\mathrm{H}]^{+} .537[\mathrm{M}+\mathrm{H}-130]^{+} .535$ $[\mathrm{M}+\mathrm{H}-132]^{-}, 405[\mathrm{M}+\mathrm{H}-130-132]^{+}, 2+3$ [M+H-130-132-162], 131 [cinnamoyl] $]^{-}{ }^{1} \mathrm{H}$ NMR (400 MHz $\left.\mathrm{CD}_{3} \mathrm{OD}\right)$ and ${ }^{13} \mathrm{C}$ NMR (100
$\mathrm{MHz}, \mathrm{CD}_{3} \mathrm{OD}$ ) data see Table 1

(E)-Resveratol 3-O- $\alpha$ - $D$-glucopyramoside (2): White acicular crystals $(\mathrm{MeOH})$. mp. 202-205 ${ }^{\circ} \mathrm{C}$; HR-FAB-MS (positive mode) $m z: 391.1384\left[\mathrm{M}+\mathrm{H}^{+}\right.$(calculated for $\mathrm{C}_{2} \mathrm{H}_{33} \mathrm{O}$. 391.1393 ); FAB-MS (positive mode) mz: $391[\mathrm{M}+\mathrm{H}]^{+} .229$ [M+H-162] $^{+}$: ${ }^{\mathrm{l}} \mathrm{H} \mathrm{NMR}\left(400 \mathrm{MHz}, \mathrm{CD}_{5} \mathrm{OD}\right) \delta_{\mathrm{H}}: 7.38(2 \mathrm{H} . \mathrm{d}, J=8.6 \mathrm{~Hz}, \mathrm{H}-2$ ' and $\left.6^{\prime}\right), 6.99(\mathrm{lH}, \mathrm{d} . J=16.1 \mathrm{~Hz}, \mathrm{H}-8), 6.85(\mathrm{lH}, \mathrm{d} . J=16 . \mathrm{l}$ Hz. H-7). $6.78\left(2 \mathrm{H}, \mathrm{d}, J=8.6 \mathrm{~Hz} . \mathrm{H}-3^{\prime}\right.$ and $\left.5^{\prime}\right) .6 .71$ (1H. br s, $\mathrm{H}-2$ ). 6.57 (lH. br s. H-6). 6.39 (lH. br s. H-4). 5.33 (lH. d. $J$ $=3.5 \mathrm{~Hz}, \mathrm{H}-1$ "). 3.68-3.22 (6H, m. H-2", 3". 4", 5". 6"a.6"b): ${ }^{12} \mathrm{C}$ NMR (100 MHz. CD $\mathrm{CDD}_{3} \mathrm{OD} \delta_{6}: 159.4(\mathrm{C}-3), 158.8(\mathrm{C}-5)$, $158.3\left(\mathrm{C}-4^{\prime}\right) .140 .6(\mathrm{C}-1), 128.8(\mathrm{C}-8) .128 .5\left(\mathrm{C}-\mathrm{l}^{\prime}\right) .127 .9$ $\left(\mathrm{C}-2^{\prime}\right.$ and $\left.6^{\prime}\right) .126 .1(\mathrm{C}-7), 115.5\left(\mathrm{C}-3^{\prime}\right.$ and $\left.5^{\prime}\right) .107 .5(\mathrm{C}-6)$. $105.2(\mathrm{C}-4), 103.4(\mathrm{C}-2), 98.3\left(\mathrm{C}-\mathrm{I}^{\prime \prime}\right), 73.8(\mathrm{C}-5 "), 73.3$ (C-3"), $72.4\left(\mathrm{C}-2^{\prime \prime}\right), 70.3\left(\mathrm{C}-4^{\prime \prime}\right) .61 .2$ (C-6").

(E)-3-Hydroxyanethole 3-O-B-D-glucopyranoside (3): White amorphous powder $(\mathrm{MeOH}),[\alpha]_{D}^{22}-26.5^{2}(c) 0.35$. $\mathrm{MeOH}$ ): lit. $^{39}:-28^{\circ}$ (c 0.40. MeOH): HR-FAB-MS (positive mode) $m: z: 327.1439\left[\mathrm{M}+\mathrm{H}^{-}\right.$(calculated for $\mathrm{C}_{16} \mathrm{H}_{23} \mathrm{O}$, $327.1444) .165[\mathrm{M}+\mathrm{H}-162]^{-}:{ }^{1} \mathrm{H}$ NMR (400 MHz. CD $\left.{ }_{3} \mathrm{OD}\right) \delta_{\mathrm{H}}$ : $7.32(\mathrm{lH}, \mathrm{d} . J=1.5 \mathrm{~Hz}, \mathrm{H}-2) .7 .08(\mathrm{lH}$. dd $J=8.0$ and $1.5 \mathrm{~Hz}$. $\mathrm{H}-6) .6 .92(\mathrm{lH}, \mathrm{d}, J=8.0 \mathrm{~Hz}, \mathrm{H}-5) .6 .40(1 \mathrm{H}, \mathrm{d} . J=16.0 \mathrm{~Hz}$. $\left.\mathrm{H}-1^{\prime}\right) .6 .23\left(1 \mathrm{H}, \mathrm{dq} . J=16.0\right.$ and $\left.6.1 \mathrm{~Hz} . \mathrm{H}-2^{\prime}\right) .4 .92(1 \mathrm{H}, \mathrm{d} . J$ $\left.=7.5 \mathrm{~Hz} . \mathrm{H}-\mathrm{l}^{\prime \prime}\right) .3 .84\left(\mathrm{lH}\right.$, dd $J=11.9$ and $\left.1.8 \mathrm{~Hz} . \mathrm{H}-6^{\prime \prime} \mathrm{a}\right)$. 3.75 (3H.s. $\left.\mathrm{CH}_{3} \mathrm{O}\right), 3.61$ (1H. dd. $J=4.6$ and $\left.11.9 \mathrm{~Hz} . \mathrm{H}-6^{\prime \prime} \mathrm{b}\right)$, $3.38\left(\mathrm{lH}\right.$. dd $J=7.5$ and $\left.9.0 \mathrm{~Hz}, \mathrm{H}-2^{\prime \prime}\right) .3 .34$ ( $\left.\mathrm{lH}, \mathrm{m} . \mathrm{H}-3^{\prime \prime}\right)$. $3.26\left(1 \mathrm{H} . \mathrm{m} . \mathrm{H}-4^{\prime \prime}\right), 3.2+\left(1 \mathrm{H}, \mathrm{m}, \mathrm{H}-5^{\prime \prime}\right) .1 .68(3 \mathrm{H} . \mathrm{d} . j=6.1$ $\left.\mathrm{Hz} . \mathrm{H}-3^{\prime}\right) ;{ }^{12} \mathrm{C}$ NMR (100 MHz. $\left.\mathrm{CD}_{5} \mathrm{OD}\right) \delta_{0} \cdot 149.8$ (C-4), $148.6(\mathrm{C}-3), 132.1(\mathrm{C}-1), 131.8\left(\mathrm{C}-\mathrm{l}^{\prime}\right), 124.5\left(\mathrm{C}-2^{\prime}\right), 121.6$ (C-6). $11+.5(\mathrm{C}-2), 113.5(\mathrm{C}-5), 102.1\left(\mathrm{C}-1^{\prime \prime}\right), 78.6\left(\mathrm{C}-3^{\prime \prime}\right)$, $77.8\left(\mathrm{C}-5^{\prime \prime}\right) .74 .5\left(\mathrm{C}-2^{\prime \prime}\right) .71 .3\left(\mathrm{C}-4^{\prime \prime}\right) .61 .9\left(\mathrm{C}-6^{\prime \prime}\right) .56 .4$ $\left(\mathrm{OCH}_{3}\right), 18.6\left(\mathrm{C}-3^{\prime}\right)$; Significant HMBC $\left({ }^{2-3} J_{\mathrm{CH}}\right)$ and ROESY correlations: $\mathrm{H}-2 / \mathrm{C}-3, \mathrm{C}-4, \mathrm{C}-6$ and $\mathrm{C}-\mathrm{l}^{\prime}: \mathrm{H}-5 / \mathrm{C}-1, \mathrm{C}-3$ and $\mathrm{C}-4: \mathrm{H}-6 / \mathrm{C}-2, \mathrm{C}-4$ and $\mathrm{C}-\mathrm{I}^{\prime}: \mathrm{H}-\mathrm{I}^{\prime} / \mathrm{C}-2$. C-6. C-2' and $\mathrm{C}-3^{\prime}: \mathrm{H}-$ $2 / \mathrm{C}-1 . \mathrm{C}-\mathrm{I}^{\prime}$ and $\mathrm{C}-3^{t}: \mathrm{H}-3^{\prime} / \mathrm{C}-\mathrm{I}^{\prime}$ and $\mathrm{C}-2^{\prime}: \mathrm{H}-\mathrm{OCH}_{3} / \mathrm{C}-4 ; \mathrm{H}-1^{\prime \prime} /$ $\mathrm{C}-3: \mathrm{H}-\mathrm{l}^{\prime \prime} / \mathrm{H}-2$ and $\mathrm{H}-3^{\prime \prime}: \mathrm{H}-2 / \mathrm{H}-2^{\prime}: \mathrm{H}-5 / \mathrm{H}-\mathrm{OCH}_{2}$

Orcinol $O-\beta-D$-glucopyranoside (4): White amorphous powder $(\mathrm{MeOH}), \mathrm{mp} .131-132^{\circ} \mathrm{C}$. lit. ${ }^{+2}: 132-133^{\circ} \mathrm{C}$ : FAB-MS (positive node) $m: 287[\mathrm{M}+\mathrm{H}]^{-}, 125[\mathrm{M}+\mathrm{H}-162]^{+} ;{ }^{1} \mathrm{H}$ NMR (400 MHz, $\left.\mathrm{CD}_{3} \mathrm{OD}\right) \delta_{\mathrm{H}}: 6.38(1 \mathrm{H}$, br s, H-2), $6.26(1 \mathrm{H}$, br s, $\mathrm{H}-4) .6 .25$ (lH. br s. H-6). $4.9 \mathrm{l}$ (lH. d. $J=7.4 \mathrm{~Hz}$. H-l'). 3.78 (1H. dd, $J=11.8$ and $1.6 \mathrm{~Hz}$. H-6'a). 3.59 (1H, dd, $J=4.5$ and $11.8 \mathrm{~Hz}, \mathrm{H}-6 \mathrm{~b}), 3.48(\mathrm{lH}, \mathrm{m}, \mathrm{H}-4), 3.46(\mathrm{lH}, \mathrm{dd}, J=7.4$ and $\left.9.0 \mathrm{~Hz} . \mathrm{H}-2^{\prime}\right), 3.42\left(1 \mathrm{H}, \mathrm{m} . \mathrm{H}-3^{\prime}\right) .3 .22\left(1 \mathrm{H}, \mathrm{m}, \mathrm{H}-5^{\prime}\right) .2 .16$ $\left(3 \mathrm{H}, \mathrm{s}, \mathrm{CH}_{3}\right):{ }^{13} \mathrm{C} \mathrm{NMR}\left(100 \mathrm{MHz}, \mathrm{CD}_{3} \mathrm{OD}\right) d \mathrm{C}: 159.1(\mathrm{C}-1)$. $158.2(\mathrm{C}-3), 1+1.2(\mathrm{C}-5), 111.2(\mathrm{C}-6), 109.8(\mathrm{C}-4), 103.4$ $(\mathrm{C}-2) .101 .8\left(\mathrm{C}-1^{\prime}\right) .78 .3\left(\mathrm{C}-3^{\prime}\right) .78 .1\left(\mathrm{C}-5^{\prime}\right), 74.9\left(\mathrm{C}-2^{\prime}\right) .71 .8$ $\left(\mathrm{C}-4^{\prime}\right), 62.7\left(\mathrm{C}-6^{\prime}\right), 21.8\left(\mathrm{CH}_{3}\right)$

Acid hydrolysis of compounds 1 and detemination of the absolute configuration of the monosacchaide. Compounds 1 (10 $\mathrm{mg}$ ) were dissolved in $\mathrm{MeOH}(10 \mathrm{~mL})$ and $2 \mathrm{~N} \mathrm{HCl}(5$ $\mathrm{mL}$ ), respectively. The nuxtures were refluxed with magnetic stirring in a water bath at $90^{\circ} \mathrm{C}$ for $2 \mathrm{~h}$. After cooling. the reaction mixture was diluted with $5 \mathrm{~mL}$ of $\mathrm{H}_{2} \mathrm{O}$. then extracted twice with EtOAc. The EtOAc phase was evaporated and trans-cinnanic acid was identified by TLC with standard samples. The aqueous layer were neutralized by passing through an ion-exchange resin (Amberlite MB-3) column eluted with 
$\mathrm{H}_{2} \mathrm{O}$. then concentrated and dried to furnish a monosaccharide residue. The residue was trimethylsilylated with $\mathrm{I}$-(trimethylsilyl)-imidazole at ambient temperature for $2 \mathrm{~h}$. After the excess reagent was decomposed with $\mathrm{H}_{2} \mathrm{O}$, the mixture was partitioned between $n$-hexane $(2 \mathrm{~mL})$ and $\mathrm{H}_{2} \mathrm{O}(2 \mathrm{~mL})$. and the $n$-hexane extract was concentrated and analyzed by $\mathrm{GC}$ under the following conditions: HP-5 MS fused silica capillary column $(30 \mathrm{~m} \times 0.25 \mathrm{~mm}$. film thinkness $0.25 \mu \mathrm{m})$, column temperature at $230^{\circ} \mathrm{C}$. injection temperature at $250^{\circ} \mathrm{C}$. $\mathrm{N}_{2}$ as carrier gas. In the acid hydrolysate of 1, D-glucose and $D$-apiose were confirmed by comparison of the retention times of their derivatives with those of $D$-glucose $(22.8 \mathrm{~min}$ ). $L$-glucose (21.9 min). $D$-apiose (11.6 min), $L$-apiose (10.1 min). respectively

Acknow ledgments. This work was financially suported by the Postgraduates' Innovative Education Program (NWU Graduate Cross-discipline Funds. No. 08YJC20) of Northwest University, P. R. China. The authors are gratefil to Prof. Qi-xiu Zhu and Prof. Fan-zhi Zhao of the Analytical Center of Lanzhou University for NMR and MS measurements

\section{References}

1. Delectis Florae Reipublicae Popularis Sinicae, Agendae Acadeniae Sinicae Edits, Flora Reipublicae Popularis Sinicae: Science Press: Beijing, China, 1980; Vol. 54, p 104.

2. Na, N.; Xiangqian, L. Chin. Tradit. Herb. Dnigs 2006, 37, 1895.

3. Nishibe, S.; Kinoshita, H.: Takeda, H.: Okano, G. Chem. Pham. Bull. 1990, 38,1763 .

4. Fujikawa, T: Yamaguchi, A.; Morita, I:; Takeda, H.; Nishibe, S. Biol. Pham. Bull. 1996, 19, 1227.

5. Park, S.-Y: Yook, C.-S.: Nohara, T. Tetmhedron Lett. 2001, 42, 2825

6. Yook, C.-S.: Kinn, J-H.: Hulu1, D.-R.: Nohara, T.: Chang, S.-Y. Photochentistry 1998, 49,839.

7. Wanjun, Z. Recont Chinese Wooly Plants: Chinese Forestry Press: Beijung, China, 1985: Vol. 2, p 1777.

8. Zhongli, W.: Linde, L.: Guowei, T., Tiaheng, S. Chin Biodiver. $1997,5,251$

9. Jinuing, Z.; Yingcai, Z.; Yukun, R. J. Ningxia Agric. Coll. 2000 , 21,81 .

10. Lipin, L. Zimuling Hoody Flora: Lanzhou University Press: Lanzhou, China, 1997: p 215

11. Xiaoqiang, G. Ganst Agric. Sci. Technol. 2004, 4t, 49

12. Lin, C. C. Huang, P. C. Phyther. Res. 2000, $1+489$.

13. Yi, T. M.: Kinn, M. S.; Seo, S. W.: Lee, K. N.; Yook, C. S.; Kin, H. M. Clin. Chim. Acta 2001, 32, 163
14. Deyama, T. Nishibe, S.; Nakazawa, Y. Acta Phomacol. Sin. 2001, 22, 1057.

15. Ping, W.; Gaomai, Y. Chin. Tradit. Herb. Drths $1989,20,8$

16. Ping, W J. Chin. Mater. Med. 1988, 11, 48 .

17. Miyakoshi, M.; Ida, Y.; Shoji, J. Phvtochemistry 1993, 34, 1599.

18. Chunming, L.; Jimin, Z:; Hongmei, L.; Fengrui, S. Chem. Res. Chin. Lin. 2007, 23, 233.

19. Na, M. K.; Oh, W. K.; Kim, Y. H.; Cul, X. F.; Kim, S. H.; Kim, B. Y.; Aln, J. S. Bioorg Med. Chem Letr. 2006, 16, 3061.

20. Mingquan, G.: Fengrui, S.: Zhiqiang. L.; Shuying. L. Anal. Chim. Acta 2006, 557, 198 .

21. Ban, H. S.; Lee, S.; Kim, Y. P.; Yamaki, K.; Shin, K. H.; Ohuchi, K. Biachem. Phamacol $2002,64,1345$.

22. Kashiwada, Y.; Nonaka, G. I.; Nishioka, I. Chent Pham. Bull. $1984,32,3501$.

23. Karl, C.; Muller, G.; Pedersen, P. A. Phytochemistry 1976, 15, 1084.

24. Aritomi, M. Chem. Pham Bull. 1963, 11, 1125.

25. Xiao, K. Xuan, L.; Xu, Y: Bai, D. J. Not. Prod. 2000, 63, 1373.

26. Anjaneyulu, A. S. R.: Reddy, R. A. V.; Reddy, D. S. K.; Ward, R. S.: Adhikesavalu, D.; Cameron, S. T. Tetrahedron 1984, 40 , 4245 .

27. Zhenlu, S.; Guanglan, Z.; Xuanzhen, J. Indian J. Chem. Sect-B 2002, H1, 2395.

28. Wanjala. C. C. W.: Majinda, R. R. T. Fitoterapia 2001, 72, 649

29. Gromova, A. S.: Tyukavkina, N. A.; Lutskii, V. I:; Kalabin, G. A.: Kushnarev, D. F. Chem. Nat. Compd. 1975, 11, 715.

30. Takahashi, H.; Hirata, S.; Hiroyuki, M.; Fukuyama, Y. Phtochentistry 2001, 56,875.

31. Hara, S.; Okabe, H.; Mihashi, K. Chent. Phom. Bull. 1987, 35, 501

32. Yang. H.: Sung. S. H.: Kim. Y. C. J. Kat. Prod. 2005. 68, 101

33. Dorman, D. E.; Roberts, T. D. J. Am. Chem. Soc. 1970, 92, 1355.

34. Kamerling, T. P.; De Bie, M. I. A.; Vliegenthart, J. F. G. Tetrohedron 1972, 28,3037.

35. Yamasaki, K.: Kasai, R.: Masaki, Y.: Okihara, M.: Tanaka, O.; Oshio, H.: Takagi, S.: Yamaki, M.; Masuda, K.: Nonaka, G.; Tsuboi, M.: Nishioka, I. Tetrahedron Lett. 1977, 18, 1231.

36. Agrawal, P. K.; Bansal, M. C. Carbon-13 M.R of Flovonoids; Elsevier: Amsterdam, 1989; 313

37. Mattivi, F.: Reniero, F.: Korhammer, S. J. Agric. Food Chem $1995,43,1820$

38. Heevoung. S.: Wanpyo. H.: Yonghyun. A. Bull Korean Chem. Sac. $2003,24,1680$.

39. Fujimatu, E.; Ishikawa, T.; Kitajima, J. Phvtochentistrv 2003, 63,609

40. Junping, X.: Qinvi, D. Chin. Tradit. Herb. Dngs 1986.17.8.

41. Changyiang, C., Wei, N.: Wenli, M. Acta Bot. Fmman 1999, 21 , 521

42. Ning, L.; Youxing, Z; Aiqun, T.; Yuqing, L.; Jun, Z Not. Prod. Res. Dev, 2003, 15, 208 